\title{
Epithelial neutrophil-activating peptide-78 recruits neutrophils into pleural effusion
}

\author{
G-N. Liu*, H-Z. Shi*, Z-H. Xie*, H-H. Shen\#, H-Q. Huang* , J-M. Deng*, \\ Q-L. Liang* and Y-B. Wu*
}

ABSTRACT: The aim of this study was to investigate the presence of epithelial neutrophilactivating peptide (ENA)-78 in pleural effusions, as well as the chemoattractant activity of pleural ENA-78 on neutrophils.

Pleural effusion and serum samples were collected from 75 patients who presented to the respiratory institute (19 with malignant pleural effusion, 21 with tuberculous pleural effusion, 18 with infectious pleural effusion and 17 with transudative pleural effusion). The concentrations of ENA-78, myeloperoxidase and neutrophil elastase were determined, and the chemoattractant activity of ENA-78 for neutrophils both in vitro and in vivo was also observed.

The concentrations of ENA-78, myeloperoxidase and neutrophil elastase in infectious pleural effusion were significantly higher than those in malignant, tuberculous and transudative groups, respectively (all $p<0.01$ ). Infectious pleural fluid was chemotactic for neutrophils in vitro and antiENA-78 antibody could partly inhibit these chemotactic effects. Intrapleural administration of ENA-78 produced a marked progressive influx of neutrophils into pleural space.

Compared with noninfectious pleural effusion, ENA-78 appeared to be increased in infectious pleural effusion. Our data suggested that ENA-78 was able to induce neutrophil infiltration into pleural space and might be responsible for pleural neutrophil degranulation.

KEYWORDS: Infections, inflammatory cell, neutrophil migration, pleural effusion

$\mathbf{T}$ he inflammatory process results in increased pleural vascular permeability, leading to the accumulation of fluid enriched in proteins and to the recruitment of cells with the development of exudative pleural effusion (PE) [1]. PE is characterised by the presence of specific subsets of leukocytes [1, 2] which, together with pleural mesothelial cells, contribute to the local production of cytokines and chemokines [3, 4]. Analysis of malignant and tuberculous PEs usually shows a lymphocytic preponderance; whereas infectious PEs, including empyema and parapneumonic effusion, are typically associated with an influx of neutrophils $[5,6]$.

Neutrophils are recruited to sites of inflammation by a variety of chemoattractants, including complement factors (C5a), interleukin (IL)-8, arachidonic acid metabolites (leukotriene $\mathrm{B}_{4}$ ), and infectious peptides ( $N$-formyl-methionylleucyl-phenylalanine), which are generated and released locally at sites of injured tissue. Neutrophils release reactive oxygen and nitrogen species into the extracellular milieu, in addition to releasing their granular content, which contains several serine and neutral proteases that can produce tissue injury [7]. Chemokines are potent proinflammatory molecules, and their regulation must therefore be tightly controlled [8]. One of these, a CXC chemokine, is epithelial neutrophilactivating peptide (ENA)-78 (also named CXCL5), which contains a Glu-Leu-Arg motif essential for neutrophil-stimulating activity [9, 10]. ENA-78 has been implicated in the pathogenesis of a number of pulmonary diseases [1113]. The present study was performed to determine total and differential leukocyte counts and the concentrations of ENA-78 and two indicators of neutrophil degranulation (myeloperoxidase (MPO) and neutrophil elastase (NE)) in pleural fluid and serum of patients with PEs of various aetiologies, in order to: 1) determine whether PE ENA-78 is produced in the pleural space; 2) establish the relationship between this chemokine to the neutrophil number and activation state in PE; and 3) explore the chemoattractant activity of ENA-78 on neutrophils.

\section{MATERIALS AND METHODS \\ Subjects}

A prospective design study was performed in our institute of respiratory diseases (First Affiliated Hospital, Guangxi Medical University, Guangxi, China) from October, 2006 to March, 2008. The
AFFILIATIONS

*Institute of Respiratory Diseases, First Affiliated Hospital, Guangxi Medical University, Nanning, Guangxi, and

${ }^{\text {\#} D e p t ~ o f ~ R e s p i r a t o r y ~ D i s e a s e, ~}$ Second Hospital of Medical School of Zhejiang University, Hangzhou, Zhejiang, China.

CORRESPONDENCE

H-Z. Shi

Institute of Respiratory Diseases, First Affiliated Hospital

Guangxi Medical University Nanning 530021

Guangxi

China

E-mail: shihuanzhong@sina.com

Received:

July 222008

Accepted after revision:

Nov 062008

First published online:

Dec 012008 
study protocol was approved by our institutional review board for human studies and informed consent was obtained from all subjects. 75 consecutive patients with PE of various causes were recruited in the present study.

Malignant PE was collected from 19 patients (aged 34-74 yrs) with newly diagnosed lung cancer with PE. Histologically, 14 cases were adenocarcinoma and 5 were squamous cell carcinoma. A diagnosis of malignant PE was established by demonstration of malignant cells in PE and/or on closed pleural biopsy specimen.

21 patients (aged 17-68 yrs) were proven to have tuberculous PE, as evidenced by: a compatible clinical history associated with presence of acid-fast bacilli in PE specimen or by demonstration of granulomatous pleurisy on closed pleural biopsy specimen in the absence of any evidence of other granulomatous diseases; an exudative lymphocytic effusion with an adenosine deaminase level $>40 \mathrm{U} \cdot \mathrm{L}^{-1}$, along with a positive purified protein derivative skin test result and the exclusion of any other potential causes of pleurisy; or following antituberculosis chemotherapy, the resolution of PE and clinical symptoms was observed.

18 PE patients (aged 16-57 yrs) were classified as infectious PE (including 16 empyema and two parapneumonic effusion). Empyema was defined as an effusion that met one or more of the following criteria: purulent fluid on macroscopic examination, positive Gram stain and/or growth of organisms in culture, and $\mathrm{PE}$ with $\mathrm{pH}<7.2$ or glucose $<3.3 \mathrm{mmol} \cdot \mathrm{L}^{-1}$ in association with pneumonia. Parapneumonic effusion was defined as those with a glucose concentration $>3.3 \mathrm{mmol} \cdot \mathrm{L}^{-1}$ and $\mathrm{pH}>7.2$, and no organisms seen on Gram stain or found on PE culture in a patient with pneumonia.

17 patients (aged 17-70 yrs) with PE were classified as transudates on the basis of Light's criteria [14].

Patients were excluded if they had received any invasive procedures directed into the pleural cavity or if they had suffered chest trauma within 3 months prior to hospitalisation or had a PE of undiagnosed cause. At the time of sample collection, none of the patients had received any antituberculosis therapy, anticancer treatment, corticosteroids or other nonsteroidal anti-inflammatory drugs.

\section{Sample collection and processing}

PE samples were collected from each subject in heparin-treated tubes, using a standard thoracocentesis technique within $24 \mathrm{~h}$ after hospitalisation. $10 \mathrm{~mL}$ of venous blood was drawn simultaneously for obtaining sera. The PE specimens were immersed in ice immediately and were then centrifuged at $1,200 \times g$ for $5 \mathrm{~min}$. The cell-free supernatants of PE and serum were frozen at $-80^{\circ} \mathrm{C}$ immediately after centrifugation, for later determining concentrations of ENA-78, MPO and NE. Total and differential cell counts, and detections of concentrations of pleural protein, glucose, lactate dehydrogenase and adenosine deaminase, were performed in addition to cytological and microbiological examination of pleural fluid. A pleural biopsy was performed when the results of pleural fluid analysis were suggestive of tuberculosis or malignancy.

\section{Measurement of ENA-78, MPO and NE}

The concentrations of ENA-78, MPO and NE in both PEs and sera were measured by sandwich ELISA kits according to the manufacturers' protocols. The ELISA kits for ENA-78 and MPO were purchased from R\&D Systems Inc. (Minneapolis, MN, USA), and the ELISA kits for NE were purchased from Cell Sciences ${ }^{\circledR}$ (Canton, MA, USA). All samples were assayed in duplicate. The lower detection limits were: $15 \mathrm{ng} \cdot \mathrm{L}^{-1}$ (ENA78); $0.1 \mu \mathrm{g} \cdot \mathrm{L}^{-1}(\mathrm{MPO})$; and $0.4 \mu \mathrm{g} \cdot \mathrm{L}^{-1}(\mathrm{NE})$.

\section{Neutrophil chemotaxis assay}

Human neutrophils were prepared from peripheral blood by Ficoll-Hypaque density gradient in $0.9 \%$ saline (Sigma Chemical Co., St. Louis, MO, USA). Neutrophils were separated from erythrocytes by hypotonic lysis and then suspended in Hanks' balanced salt solution (HBSS) with calcium/magnesium (Gibco; Grand Island, NY, USA) at $2.0 \times 10^{6} \mathrm{cells} \cdot \mathrm{mL}^{-1}$ with $>95 \%$ viability by trypan blue exclusion. $200 \mu \mathrm{L}$ of the neutrophil suspension $\left(1.0 \times 10^{6}\right.$ cells $\cdot \mathrm{mL}^{-1}$ in RPMI-1640 media) were loaded into the upper well of a 24-well Transwell chamber (Costar, Corning NY, USA) while $30 \mu \mathrm{L}$ pleural fluid from five patients with malignant $\mathrm{PE}$, five with tuberculous $\mathrm{PE}$, five with empyema or five with transudates, was placed in the bottom chamber. The two wells were separated by a polycarbonate filter paper with a pore size of $5 \mu \mathrm{m}$ and the chamber was incubated at $37^{\circ} \mathrm{C}$ for $45 \mathrm{~min}$. At the end of incubation, the filter was fixed, stained and mounted on a glass microscope slide. Migration was assessed by counting the number of cells that had migrated beyond a certain depth into the filter $(50 \mu \mathrm{m})$. To correct for interdonor variation, migration data of test samples were compared with their corresponding control values (HBSS alone) and expressed as a percentage above the control value. To demonstrate that ENA-78 was responsible for neutrophil migration, blocking experiments were performed by mixing the PE with $10 \mu \mathrm{g} \cdot \mathrm{mL}^{-1}$ of anti-ENA-78 monoclonal antibody $(\mathrm{mAb})$ or immunoglobulin $\mathrm{G}_{2 \mathrm{a}}$ irrelevant isotype control (R\&D System Inc.).

\section{Effects of intrapleural injected ENA-78 on neutrophil recruitment}

After an additional study protocol was approved by our institutional review board and informed consent was obtained from the subjects studied, a total of 10 patients with tuberculous PE were included in this section of the study. Immediately after collection of PE samples, $10 \mu \mathrm{g}$ of recombinant human ENA-78 (R\&D Systems Inc.) in vehicle $(0.1 \%$ human serum albumin in $0.9 \%$ saline) was injected into the pleural space of five patients and vehicle only was injected into the pleural space of the other five patients. Intrapleural injection of ENA-78 or vehicle was randomised. The dose of ENA-78 was based upon a preliminary study involving two PE patients. PE collection for determining neutrophil number was repeated 6, 12 and $24 \mathrm{~h}$ after the injection of ENA-78 or vehicle.

\section{Statistical analysis}

Data were expressed as mean \pm SEM or median (25th-75th percentile). Comparisons of the data between different groups were performed using Kruskal-Wallis one-way ANOVA on ranks. For the levels of ENA-78, MPO and NE in PE and in corresponding serum, paired data comparisons were made using a Wilcoxon signed-rank test. The correlations between 
variables were determined by Spearman's rank correlation coefficients. The effects of intrapleural injected ENA-78 or vehicle on neutrophil recruitment were compared through one-way repeated-measures ANOVA. Analysis was completed with SPSS version 14.0 Statistical Software (Chicago, IL, USA), and a p-value $<0.05$ was considered significant.

\section{RESULTS}

\section{Characteristics in PEs}

Cytological characteristics in PEs are illustrated in table 1. Subjects with lung cancer showed a large proportion of lymphocytes and macrophages in PE. Importantly, on cytological examination, malignant cells were found in 13 subjects. Subjects with tuberculosis showed a marked elevation of total cell counts and a large proportion of these cells were lymphocytes, with some neutrophils and macrophages. Absolute lymphocyte counts evidenced the highest values in tuberculous PE, showing a significant increase in comparison with each of the PEs induced by the other causes (all $\mathrm{p}<0.05$ ). Also, as expected, total cell counts in infectious PE were the greatest among the four groups (all $\mathrm{p}<0.05)$ and neutrophils were the predominant cell type; significantly greater than those in the PEs with any other causes (all $\mathrm{p}<0.01$ ).

\section{Concentrations of ENA-78, MPO and NE in PEs}

As shown in table 2 and figure 1, the concentration of ENA78 in infectious PE (median 2,639.2 (25th-75th percentile, 448.8-5,014.6) $\mathrm{ng} \cdot \mathrm{L}^{-1}$ ) was significantly higher than those in malignant PE (88.0 (52.2-337.8) $\left.\mathrm{ng} \cdot \mathrm{L}^{-1}\right)$, tuberculous PE (73.0 (33.9-131.5) $\left.\mathrm{ng} \cdot \mathrm{L}^{-1}\right)$ and transudative PE (36.1 (22.374.2) $\mathrm{ng} \cdot \mathrm{L}^{-1}$ ), all with $\mathrm{p}<0.01$. It should be mentioned that, although ENA-78 concentration in infectious PE was much higher than those in the other PEs, we were unable to identify a cutoff value for ENA-78 that could be used to diagnose infectious PE.

Similar results were observed with MPO and NE studies, and the concentrations of both these enzymes changed in parallel among the groups. Additionally, as shown in table 2, the concentrations of both MPO and NE in infectious PE were significantly higher than those in the any other groups (all $\mathrm{p}<0.01)$.

\section{TABLE 1 Cytological characteristics in pleural effusions (PE)}

\section{Malignant PE}

\section{Subjects $\mathbf{n}$}

Total cell count $\times 10^{9}$ cells $\cdot \mathrm{L}^{-1}$

Differential cell counts \%

Lymphocyte $^{\#}$

Neutrophil

Macrophage ${ }^{\#}$

Mesothelial cell

Malignant cell

$\begin{array}{rccr}19 & 21 & 18 & 17 \\ 1.48 \pm 0.10 & 2.52 \pm 0.16^{\bullet} & 6.46 \pm 0.89^{+} & 0.39 \pm 0.11 \\ 46.9 \pm 1.5 & & & \\ 3.8 \pm 0.8 & 72.7 \pm 1.4 & 17.0 \pm 3.4 & 33.7 \pm 4.7 \\ 35.3 \pm 1.7 & 13.1 \pm 1.0^{+} & 77.5 \pm 3.5^{+} & 4.6 \pm 1.0 \\ 7.2 \pm 0.9 & 13.1 \pm 0.9 & 5.5 \pm 0.8 & 54.8 \pm 5.5 \\ 6.5 \pm 1.2 & 1.6 \pm 0.3^{\oplus, 5} & 0 \pm 0^{\oplus, 5} & 6.7 \pm 3.1\end{array}$

Data are presented as mean \pm SEM, unless otherwise stated. ${ }^{*}: p<0.05$ compared with one another among four groups; $": p<0.05$ compared with the transudative group $+: p<0.01$ compared with each of the other three groups; ${ }^{s}: p<0.05$ compared with the malignant group. The comparisons were determined by Kruskal-Wallis one-way ANOVA on ranks.

TABLE 2 Concentrations of epithelial neutrophil-activating peptide (ENA)-78, myeloperoxidase (MPO) and neutrophil elastase (NE) in pleural effusions and sera

Malignant PE

Subjects $\mathbf{n}$

ENA-78 $\mathbf{n g} \cdot \mathrm{L}^{-1}$

Pleural effusion

Serum

MPO $\mu \mathrm{g} \cdot \mathrm{L}^{-1}$

Pleural effusion

Serum

$\mathrm{NE} \mu \mathrm{g} \cdot \mathrm{L}^{-1}$

Pleural effusion

Serum
19

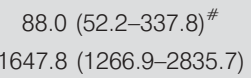

$$
\begin{gathered}
222(135.0-402.0)^{\#} \\
436(253-986) \\
83(42-173)^{\#} \\
54(14-88)
\end{gathered}
$$

Tuberculous PE

21

$73.0(33.9-131.5)^{\#}$ $1686.4(1307.9-3421.6)$

763 (563.0-1236.0) \#

$416(229-669)$

$258(96-432)^{\#}$

$42(22-77)$
Infectious PE

18

$2639.2(448.8-5014.6)^{\bullet}$ $1861.3(1060.1-2276.1)$

$36.1(22.3-74.2)^{\#}$ $1233.3(634.7-1816.9)$

$\begin{array}{cc}32011(8338-88905)^{\#,} \cdot & 87(50-137)^{\#} \\ 461(136-1340) & 412(250-882) \\ & \\ 20721(4276-57275)^{\#, \cdot} & 23(12-44) \\ 51(22-133) & 38(15-107)\end{array}$

Data are presented as median (25th-75th percentile), unless otherwise stated. PE: pleural effusion. ${ }^{*}: \mathrm{p}<0.05$ compared with the corresponding concentration in sera, determined by Wilcoxon signed-rank test; ${ }^{\circ}: p<0.01$ compared with each of the other PE groups determined by Kruskal-Wallis one-way ANOVA on ranks. 


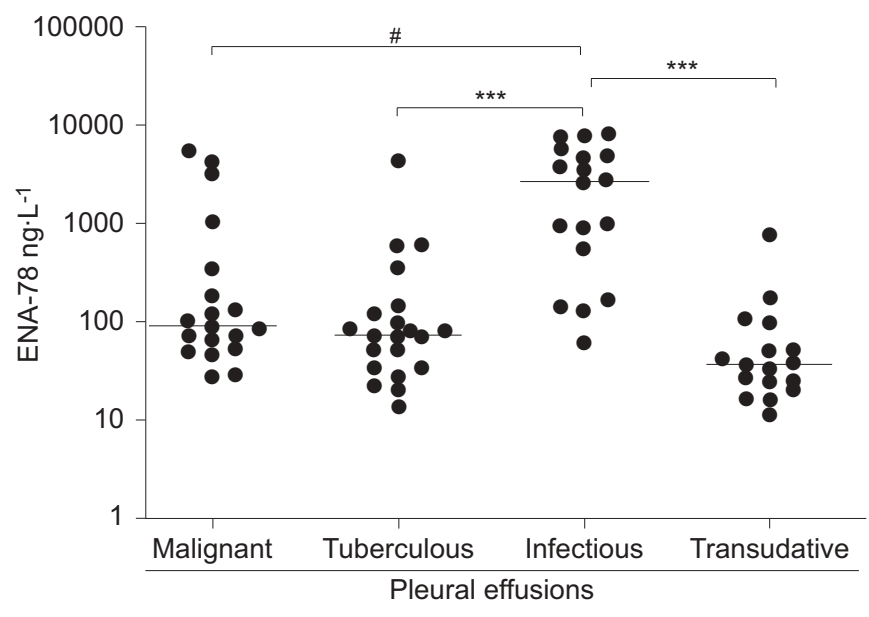

FIGURE 1. Comparison of concentrations of epithelial neutrophil-activating peptide (ENA)-78 in pleural effusions with different aetiologies. ${ }^{*}: p=0.001$; $* * *$ : $p<0.001$. Statistical analysis by Kruskal-Wallis one-way ANOVA on ranks.

The comparisons of levels of ENA-78, MPO, and NE in PEs with their corresponding concentrations in sera are also shown in table 2. The concentrations of ENA-78, MPO and NE in sera were not different from one another among the four groups (all $\mathrm{p}>0.05)$. ENA-78 concentration was much lower in PEs than in sera from patients with malignant, tuberculous and transudative PEs (all p<0.05); in contrast, ENA-78 in infectious $\mathrm{PE}$ tended to be increased when compared with the corresponding serum but the difference did not reach statistical significance $(p=0.102)$. MPO concentration was much lower in malignant and transudative PEs, and higher in tuberculous and infectious PEs than in their corresponding sera (all $\mathrm{p}<0.05)$. NE concentration in malignant, tuberculous and infectious, but not transudative, PE, was much higher than that in their corresponding serum.

\section{Correlation between ENA-78 and inflammatory cells, MPO} and NE

We noted that pleural ENA-78 levels were positively correlated with the number of neutrophils $(n=75, r=0.526$; $p<0.001$ )(fig. 2a) but not with lymphocytes $(n=75, r=0.167$; $p=0.153)$, macrophages $(n=75, r=0.022 ; p=0.854)$, mesothelial cells $(n=75, r=-0.250 ; p=0.066)$ and malignant cells $(n=13$, $\mathrm{r}=0.017 ; \mathrm{p}=0.957)$. It was further noted that the pleural ENA78 levels were positively correlated with PE MPO levels $(n=75$, $\mathrm{r}=0.714 ; \mathrm{p}<0.001)$ (fig. $2 \mathrm{~b})$ and PE NE levels $(\mathrm{n}=75, \mathrm{r}=0.739$; $\mathrm{p}<0.001$ )(fig. 2c). The correlations between pleural ENA-78 and neutrophil numbers, MPO levels and NE levels within the different diagnostic groups are shown in table 3. It was noted that PE ENA-78 levels were positively correlated with MPO levels and NE levels even when PEs with various aetiologies were analysed separately.

\section{Infectious PE was chemotactic for neutrophils}

The finding that the concentration of pleural ENA-78 correlated best with the number of neutrophils prompted us to test the chemoattractant activity of PEs on human neutrophils. The results showed that exudates, especially empyema, but not transudates, exerted a potent chemoattractant activity for neutrophils (fig. 3). In order to determine whether ENA-78
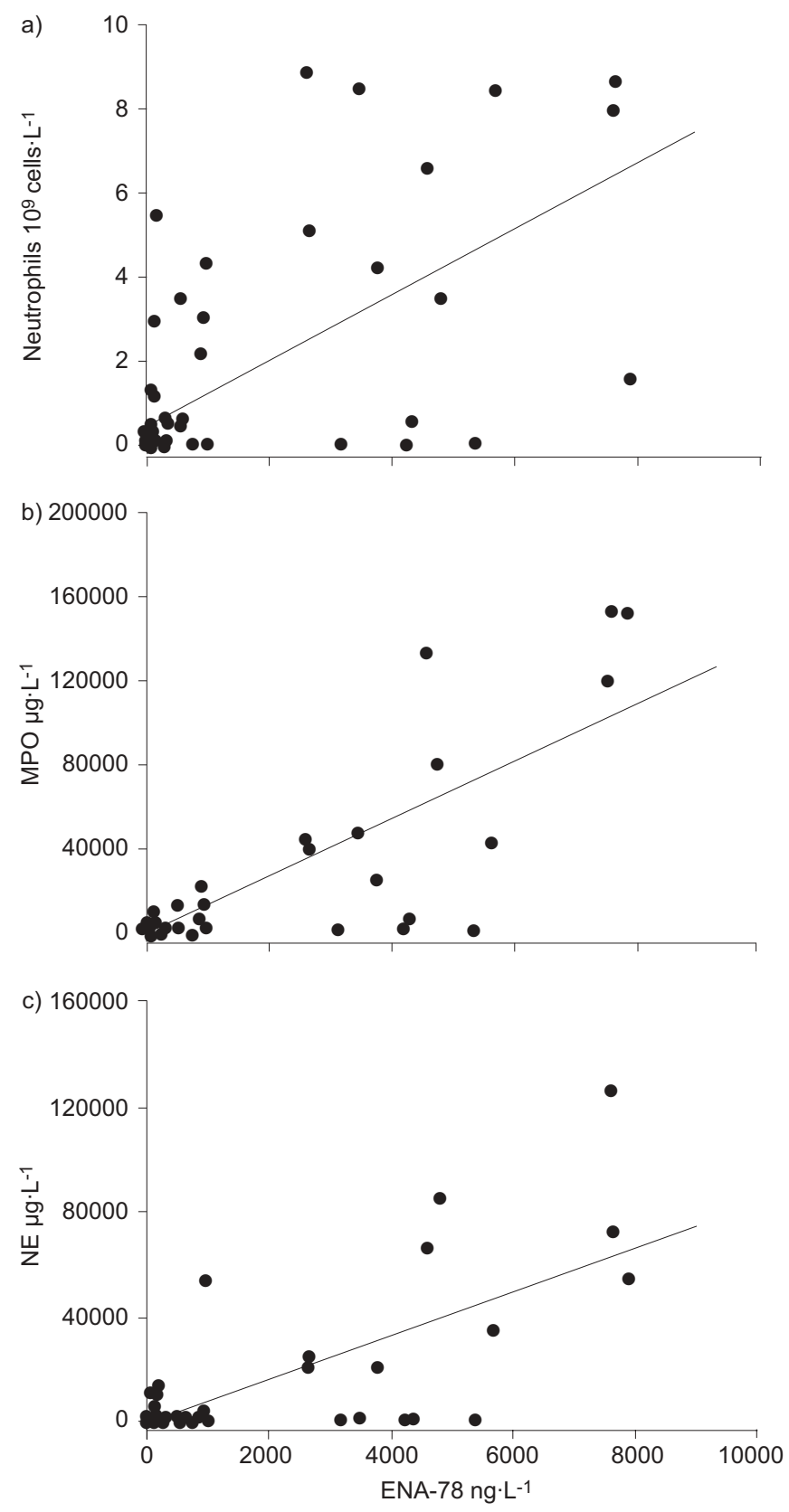

FIGURE 2. The concentrations of epithelial neutrophil-activating peptide (ENA)-78 correlated with a) neutrophil numbers $(n=75, r=0.526 ; p<0.001)$ b) myeloperoxidase (MPO; $n=75, r=0.714 ; p<0.001$ ) and c) neutrophil elastase (NE; $n=75, r=0.739 ; p<0.001$ ) in pleural effusions. Correlations were determined by Spearman's rank correlation coefficients.

was responsible for the migration of neutrophils, the ability of an anti-ENA-78 mAb to neutralise the chemoattraction of neutrophils was tested. The anti-ENA-78 mAb significantly suppressed neutrophil chemotaxis in all exudative PE groups. These data provided indirect evidence that ENA-78 was capable of inducing neutrophil recruitment into the pleural space.

\section{Recruitment of neutrophils into PE caused by ENA-78}

In order to investigate the direct chemoattractant capacity of ENA-78 to recruit neutrophils in vivo, $10 \mu \mathrm{g}$ human 


\begin{tabular}{|c|c|c|c|c|c|c|c|}
\hline ENA-78 & Subjects n & \multicolumn{2}{|c|}{ Neutrophil count } & \multicolumn{2}{|c|}{ MPO } & \multicolumn{2}{|c|}{ NE } \\
\hline Total & 75 & 0.526 & $<0.001$ & 0.714 & $<0.001$ & 0.739 & $<0.001$ \\
\hline Tuberculous PE & 21 & 0.324 & 0.152 & 0.645 & 0.002 & 0.463 & 0.035 \\
\hline Infectious PE & 18 & 0.521 & 0.027 & 0.922 & $<0.001$ & 0.723 & 0.001 \\
\hline Transudative PE & 17 & 0.136 & 0.603 & 0.574 & 0.016 & 0.914 & $<0.001$ \\
\hline
\end{tabular}

ENA: epithelial neutrophil-activating peptide; MPO: myeloperoxidase; NE: neutrophil elastase. Correlations were determined by Spearman's rank correlation coefficient.

recombinant ENA-78 were injected into the pleural space of patients with tuberculous pleurisy, and then observed the changes in neutrophil numbers. Compared with the baseline value $\left(0.38 \times 10^{9} \pm 0.06\right.$ cells $\left.\cdot L^{-1}\right)$, a significant increase in the number of neutrophils started to be observed at $6 \mathrm{~h}$ $\left(0.72 \times 10^{9} \pm 0.10\right.$ cells $\left.\cdot \mathrm{L}^{-1} ; \mathrm{p}<0.01\right)$ after intrapleural ENA-78 injection; The number of neutrophils increased with time, reaching a maximum at $12 \mathrm{~h}\left(0.82 \times 10^{9} \pm 0.10\right.$ cells $\cdot \mathrm{L}^{-1}$; $\mathrm{p}<0.01)$, and lasting for at least $24 \mathrm{~h} \quad\left(0.79 \times 10^{9}\right.$ \pm 0.10 cells $\cdot \mathrm{L}^{-1} ; \mathrm{p}<0.01$ ) (fig. 4 ). After vehicle only was injected into the pleural cavity, we did not observe increases in neutrophil counts in PE obtained at three time points when compared with baseline measurement before injection (all $\mathrm{p}>0.05)$.

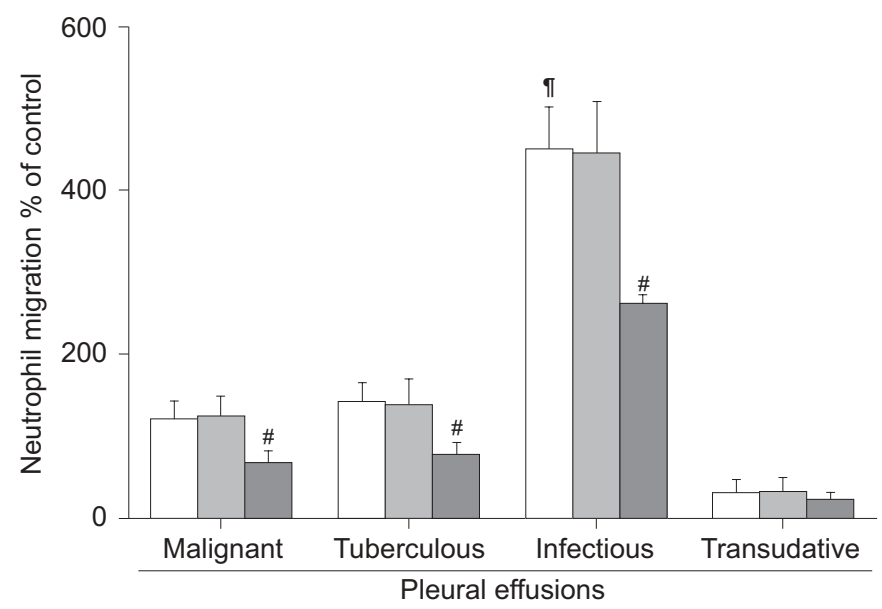

FIGURE 3. Exudative pleural effusions are chemotactic for neutrophils in vitro. Pleural effusions from patients with lung cancer $(n=5)$, tuberculous pleurisy $(n=5)$, empyema $(n=5)$ and with transudates $(n=5)$ were used to stimulate chemotaxis of peripheral blood neutrophils isolated from healthy adults. ENA-78: epithelial neutrophil-activating peptide. Data are expressed as percentage of control. $\square$ : chemotaxis in the absence of anti-ENA-78 monoclonal antibody; 1 : irrelevant isotype control; 1 : chemotaxis in the presence of anti-ENA-78 monoclonal antibody. ${ }^{\#}: p<0.01$ compared with the corresponding group without anti-ENA-78 monoclonal antibody; ": $p<0.01$ compared with each of the other pleural effusion groups. The comparisons were determined by Kruskal-Wallis one-way ANOVA on ranks.

\section{DISCUSSION}

The development of PE is often associated with an increase of inflammatory cells in the pleural space. PEs caused by diverse disease entities are usually present with the predominance of a particular type of leukocyte. Infectious PEs, including empyema and parapneumonic effusion, are typically associated with an influx of neutrophils, whereas tuberculous and malignant PEs are rich in lymphocytes $[5,6]$.

Inflammation within the pleural space could be mediated by a number of proinflammatory molecules. ENA-78 is a 78-aminoacid protein displaying the four highly conserved cysteine residues that are common feature of all CXC chemokines, including IL-8. The overall amino acid sequence identity of ENA-78 with IL-8 is only $22 \%$ [15]. ENA-78 is a potent activator of neutrophil function, inducing chemotaxis, enzyme release and rise of intracellular calcium [9] by acting via the type-B IL-8 receptors [16] and, thus, may play an important role in the recruitment of leukocytes to inflammatory lesions in a manner similar to that demonstrated for IL-8 and other neutrophil-activating proteins $[17,18]$.

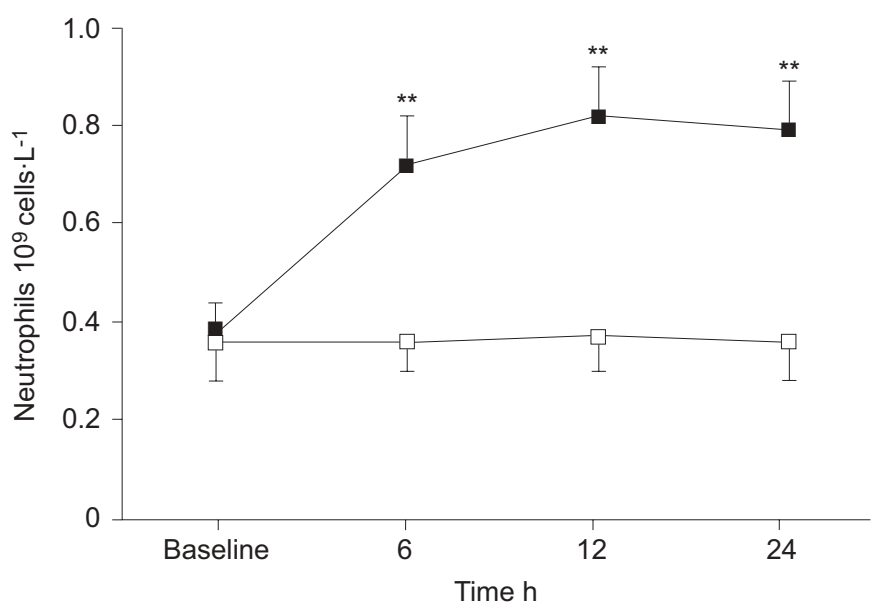

FIGURE 4. Changes of neutrophil numbers in pleural effusion from patients with tuberculous pleurisy, who were intrapleurally injected with recombinant human epithelial neutrophil-activating peptide (ENA)-78 $(\mathbf{\square}, n=5)$ or vehicle $(\square, n=5)$. Data are presented as mean \pm SEM. ${ }^{*}: p<0.01$ compared with within-group change from baseline measurements determined by one-way repeated-measures ANOVA. 
We did not identify the cellular origins of pleural ENA-78 in the present study. The primary aim of this study was to explore the presence of ENA-78 in PE, the chemoattractant activity of PE ENA-78 on neutrophils, and the relationship of ENA-78 to the activation state of neutrophils in PE. Originally, ENA-78 was characterised from the IL-1 $\beta$ - and tumour necrosis factor (TNF)- $\alpha$-stimulated alveolar type II epithelial cell line A549 [9]. Now, it is well known that ENA-78 expression is inducible by a variety of inflammation mediators, including lipopolysaccharide, IL-1 and TNF- $\alpha$, in epithelial cells [9, 19], monocytes [20, 21], platelets [22], endometrial stromal cells [23], endothelial cells [24] and macrophage [25]. In the present study, our data have shown for the first time that ENA-78 could be detected by ELISA in PEs and that the concentration of ENA-78 in infectious PE was significantly higher than those in malignant, tuberculous and transudative PEs. These findings suggested that more pleural sources of ENA-78 exist in infectious patients. Local production has been reported for some chemokines, such as IL-8 [26, 27], monocyte chemotactic peptide- $1[27,28]$ and macrophage-derived chemokine [29], in inflammatory or neoplastic pleural disorders. Likewise, PE ENA-78 can also be produced by inflammatory cells recruited into the pleural space. Interestingly, although ENA-78 concentration was much lower in PEs than in sera from patients with malignant, tuberculous and transudative PEs, ENA-78 in infectious PE tended to be increased when compared with the corresponding serum. Taken together, these results favour the concept of a local production of ENA78 in infectious $\mathrm{PE}$, rather than a passive diffusion of this chemokine from plasma to the pleural compartment. Alternatively, it was not possible for ENA-78 to exude into peripheral circulation from the pleural space in patients with malignant, tuberculous or transudative PE, since ENA-78 concentrations in these PEs were much lower than those in the corresponding sera.

The mechanism by which neutrophils infiltrate into pleural cavity has not yet been elucidated completely. The finding of higher concentration of ENA-78 in infectious PE than in any of the other groups was consistent with the massive neutrophilic infiltration of PE in this condition. If ENA-78 was the major factor contributing to neutrophil influx into the pleural space, strong correlations between PE neutrophil counts and ENA-78 would be expected. As a matter of fact, our results have demonstrated a positive correlation between neutrophil numbers and ENA-78 level, when all PEs studied were analysed together. Indeed, an in vitro migration assay in the present study further confirmed that exudative PEs could induce the migration of neutrophils and that an anti-ENA-78 $\mathrm{mAb}$ inhibited the ability of the PEs to stimulate neutrophil chemotaxis. The important findings in this study also showed that intrapleural administration of $10 \mu \mathrm{g}$ human recombinant ENA-78, not vehicle, to patients with tuberculous PE produced a marked progressive influx of neutrophils into pleural space. Therefore in the present study, we have provided the direct evidence, for the first time, that intrapleural injection of ENA78 was able to chemoattract neutrophil recruitment into the pleural space.

We noted that the effect of inhibiting ENA-78 in infectious pleural fluid only reduced neutrophil chemotaxis by $\sim 50 \%$, suggesting that ENA-78 is one of several chemokines that aid neutrophil recruitment into the pleural space. Actually, it has been reported in earlier studies that IL- 8 concentrations in infectious PE were much higher than those in PEs with any other causes, and that there was a significant positive correlation between pleural IL-8 concentrations and neutrophil counts $[27,30]$. Moreover, it has been demonstrated that IL- 8 was a major neutrophil chemotactic factor in pleural liquid of patients with empyema [27, 30]. Since ENA-78 and IL-8 exhibit different patterns of expression in cells, such as human monocytes and endothelial cells [20, 24], the comparison of chemotactic activity between ENA-78 and IL-8 needs to be established in a future study. In addition, it is likely that backup redundancy exists among the many chemokines. It is also necessary to design in vivo animal experiments using gene knockout mice or chemokine antagonists to explore such redundancy in pleural infection.

Neutrophils release reactive oxygen and nitrogen species into the extracellular milieu, in addition to releasing their granular content, which contains several serine and neutral proteases that can produce tissue injury. Among the neutrophil proteases, NE has been implicated in both chronic and acute inflammatory damage, and oxidant species, partly produced by the action of another granular enzyme, MPO, potentiate its effects [31, 32]. Therefore, it is likely that the products derived from neutrophil activation are related to the evolution of infectious PE from the noncomplicated to the complicated state. The effect of ENA-78 on neutrophil degranulation has been demonstrated in vitro $[9,33]$ and there are also data to support its occurrence in vivo [25]. In agreement with earlier studies reported by other authors, we found the highest PE concentrations of both MPO and NE in infectious PEs [34], with a profile similar to that for ENA-78. In the present study, we have shown that both MPO and NE correlated positively with ENA-78, thus suggesting a role for ENA-78 in pleural neutrophil degranulation.

It should be noted that although the ENA-78 level in infectious PE was higher than that in PE caused by the other causes, it is not possible to differentiate infectious PE from other kinds of PE due to the wide distribution and obvious overlap of these chemokine concentrations among each group.

In conclusion, compared with noninfectious PE, ENA-78 appeared to be increased in infectious PE. ENA-78 was able to induce neutrophil infiltration into pleural space and might be responsible for pleural neutrophil degranulation.

\section{SUPPORT STATEMENT}

This study was supported in part by research grants 30660064 and 30872343 from the National Natural Science Foundation of China; in part by research grant 0728137 from the Natural Science Foundation of Guangxi Zhuang Autonomous Zone; and in part by research grant 200621 from the Bureau of Health, Guangxi Zhuang Autonomous Zone, China.

\section{STATEMENT OF INTEREST}

None declared.

\section{REFERENCES}

1 Sahn SA. State of the art. The pleura. Am Rev Respir Dis 1988; 138: 184-234. 
2 Light RW. Clinical practice. Pleural effusion. N Engl J Med 2002; 346: 1971-1977.

3 Antony VB. Immunological mechanisms in pleural diseases. Eur Respir J 2003; 21: 539-544.

4 Mutsaers SE. Mesothelial cells: their structure, function and role in serosal repair. Respirology 2002; 7: 171-191.

5 Dalbeth N, Lee YC. Lymphocytes in pleural disease. Curr Opin Pulm Med 2005; 11: 334-339.

6 Hamm H, Light RW. Parapneumonic effusion and empyema. Eur Respir J 1997; 10: 1150-1156.

7 Bank U, Ansorge S. More than destructive: neutrophil-derived serine proteases in cytokine bioactivity control. J Leukoc Biol 2001; 69: 197-206.

8 Luster AD. Chemokines: chemotactic cytokines that mediate inflammation. N Engl J Med 1998; 338: 436-445.

9 Walz A, Burgener R, Car B, et al. Structure and neutrophilactivating properties of a novel inflammatory peptide (ENA-78) with homology to interleukin 8. J Exp Med 1991; 174: 1355-1362.

10 Chang MS, McNinch J, Basu R, et al. Cloning and characterization of the human neutrophil-activating peptide (ENA-78) gene. J Biol Chem 1994; 269: 25277-25282.

11 Keane MP, Belperio JA, Burdick MD, et al. ENA-78 is an important angiogenic factor in idiopathic pulmonary fibrosis. Am J Respir Crit Care Med 2001; 164: 2239-2242.

12 Wiedermann FJ, Mayr AJ, Kaneider NC, et al. Alveolar granulocyte colony-stimulating factor and $\alpha$-chemokines in relation to serum levels, pulmonary neutrophilia, and severity of lung injury in ARDS. Chest 2004; 125: 212-219.

13 Antoniou KM, Tzouvelekis A, Alexandrakis MG, et al. Different angiogenic activity in pulmonary sarcoidosis and idiopathic pulmonary fibrosis. Chest 2006; 130: 982-988.

14 Light RW, MacGregor MI, Luchsinger PC, et al. Pleural effusions: the diagnostic separation of transudates and exudates. Ann Intern Med 1972; 77: 507-513.

15 Walz A, Strieter RM, Schnyder S. Neutrophil-activating peptide ENA-78. Adv Exp Med Biol 1993; 351: 129-137.

16 Bozic CR, Gerard NP, Gerard C. Receptor binding specificity and pulmonary gene expression of the neutrophil-activating peptide ENA-78. Am J Respir Cell Mol Biol 1996; 14: 302-308.

17 Zwahlen R, Walz A, Rot A. In vitro and in vivo activity and pathophysiology of human interleukin-8 and related peptides. Int Rev Exp Pathol 1993; 34: 27-42.

18 Baggiolini M, Dewald B, Moser B. Interleukin-8 and related chemotactic cytokines: CXC and CC chemokines. Adv Immunol 1994; 55: 97-179.

19 Keates AC, Keates S, Kwon JH, et al. ZBP-89, Sp1, and nuclear factor-kappa B regulate epithelial neutrophil-activating peptide-78 gene expression in Caco-2 human colonic epithelial cells. J Biol Chem 2001; 276: 43713-43722.

20 Schnyder-Candrian S, Walz A. Neutrophil-activating protein ENA-78 and IL-8 exhibit different patterns of expression in lipopolysaccharide- and cytokine-stimulated human monocytes. J Immunol 1997; 158: 3888-3894.

21 Walz A, Schmutz P, Mueller C, et al. Regulation and function of the CXC chemokine ENA-78 in monocytes and its role in disease. J Leukoc Biol 1997; 62: 604-611.

22 Power CA, Furness RB, Brawand C, et al. Cloning of a full-length cDNA encoding the neutrophil-activating peptide ENA-78 from human platelets. Gene 1994; 151: 333-334.

23 Nasu K, Arima K, Kai K, et al. Expression of epithelial neutrophilactivating peptide 78 in cultured human endometrial stromal cells. Mol Hum Reprod 2001; 7: 453-458.

24 Imaizumi T, Albertine KH, Jicha DL, et al. Human endothelial cells synthesize ENA-78: relationship to IL-8 and to signaling of PMN adhesion. Am J Respir Cell Mol Biol 1997; 17: 181-192.

25 Halloran MM, Woods JM, Strieter RM, et al. The role of an epithelial neutrophil-activating peptide-78-like protein in rat adjuvant-induced arthritis. J Immunol 1999; 162: 7492-7500.

26 Pace E, Gjomarkaj $\mathrm{M}$, Melis $\mathrm{M}$, et al. Interleukin-8 induces lymphocyte chemotaxis into the pleural space. Role of pleural macrophages. Am J Respir Crit Care Med 1999; 159: 1592-1599.

27 Antony VB, Godbey SW, Kunkel SL, et al. Recruitment of inflammatory cells to the pleural space. Chemotactic cytokines, IL-8, and monocyte chemotactic peptide-1 in human pleural fluids. J Immunol 1993; 151: 7216-7223.

28 Yokoyama A, Kohno N, Ito M, et al. Eotaxin levels in pleural effusions: comparison with monocyte chemoattractant protein-1 and IL-8. Intern Med 2000; 39: 547-552.

29 Okamoto M, Imaizumi K, Hasegawa Y, et al. Macrophage-derived chemokine in malignant and tuberculous pleural effusions. Respirology 2007; 12: 581-584.

30 Broaddus VC, Hébert CA, Vitangcol RV, et al. Interleukin-8 is a major neutrophil chemotactic factor in pleural liquid of patients with empyema. Am Rev Respir Dis 1992; 146: 825-830.

31 Janoff A. Elastase in tissue injury. Ann Rev Med 1985; 36: 207-216.

32 Weiss SJ. Tissue destruction by neutrophils. N Engl J Med 1989; 320: 365-376.

33 Koch AE, Kunkel SL, Harlow LA, et al. Epithelial neutrophil activating peptide-78: a novel chemotactic cytokine for neutrophils in arthritis. J Clin Invest 1994; 94: 1012-1018.

34 Segura RM, Alegre J, Varela E, et al. Interleukin-8 and markers of neutrophil degranulation in pleural effusions. Am J Respir Crit Care Med 1999; 157: 1565-1572. 